Bull. Korean Math. Soc. 50 (2013), No. 2, pp. 659-665

http://dx.doi.org/10.4134/BKMS.2013.50.2.659

\title{
A NOTE ON THE TWISTED LERCH TYPE EULER ZETA FUNCTIONS
}

\author{
Yuan He AND WenPeng Zhang
}

\begin{abstract}
In this note, the $q$-extension of the twisted Lerch Euler zeta functions considered by Jang [Bull. Korean Math. Soc. 47 (2010), no. $6,1181-1188]$ is further investigated, and the generalized multiplication theorem for the $q$-extension of the twisted Lerch Euler zeta functions is given. As applications, some well-known results in the references are deduced as special cases.
\end{abstract}

\section{Introduction}

Throughout this paper, $\mathbb{Z}_{p}, \mathbb{Q}_{p}$ and $\mathbb{C}_{p}$ will denote the ring of $p$-adic rational integers, the field of $p$-adic rational numbers and the completion of the algebraic closure of $\mathbb{Q}_{p}$, respectively. Let $v_{p}$ be the normalized exponential valuation of $\mathbb{C}_{p}$ with $|p|_{p}=p^{-v_{p}(p)}=p^{-1}$. When one talks of $q$-extension, $q$ is variously considered as an indeterminate, a complex number $q \in \mathbb{C}$ or a $p$-adic number $q \in \mathbb{C}_{p}$. If $q \in \mathbb{C}$, one normally assume $|q|<1$. If $q \in \mathbb{C}_{p}$, then we normally assume $|q-1|_{p}<p^{\frac{1}{1-p}}$, so that $q^{x}=\exp (x \log q)$ for each $x \in \mathbb{Z}_{p}$. For $f \in U D\left(\mathbb{Z}_{p}, \mathbb{C}_{p}\right)=\left\{f \mid f: \mathbb{Z}_{p} \rightarrow \mathbb{C}_{p}\right.$ is the uniformly differentiable function $\}$, the $p$-adic $q$-integral (also be called as $q$-Volkenborn integration) is defined by $($ see $[6,13])$

$$
I_{q}(f)=\int_{\mathbb{Z}_{p}} f(x) d \mu_{q}(x)=\lim _{N \rightarrow \infty} \frac{1}{\left[p^{N}\right]_{q}} \sum_{j=0}^{p^{N}-1} f(j) q^{j}
$$

with $[x]_{q}=[x: q]=\left(1-q^{x}\right) /(1-q)$. For some applications of the $p$-adic $q$-integral, we infer to $[4,7,8,10,12,16,17,18,19]$.

Recently, based on the work of Kim [11], Jang [5] investigated the twisted $q$-Euler polynomials $E_{m, q, \xi}^{(-m, k)}(x)$ of order $k$ in the variable $x$ in $\mathbb{C}_{p}$ given by

Received January 5, 2012; Revised June 15, 2012.

2010 Mathematics Subject Classification. 11B68, 11S40, 05A19.

Key words and phrases. $q$-Euler number and polynomials, $q$-Euler zeta functions, Lerch type $q$-Euler zeta functions, $q$-analogue. 


$$
\begin{aligned}
E_{m, q, \xi}^{(-m, k)}(x)=\int_{\mathbb{Z}_{p}} \int_{\mathbb{Z}_{p}} & \cdots \int_{\mathbb{Z}_{p}}\left[x+x_{1}+\cdots+x_{k}\right]_{q}^{m} \xi^{x_{1}+\cdots+x_{k}} \\
& \times q^{-x_{1}(m+1)-\cdots-x_{k}(m+k)} d \mu_{-q}\left(x_{1}\right) \cdots d \mu_{-q}\left(x_{k}\right),
\end{aligned}
$$

where $k, m$ are positive integers and $\xi \in \mathbb{T}_{p}=\bigcup_{n \geq 1} \mathbb{C}_{p^{n}}$ is the locally constant space with $\mathbb{C}_{p^{n}}=\left\{\xi \mid \xi^{p^{n}}=1\right\}$ being the cyclic group of order $p^{n}$, and gave several explicit expressions of the twisted $q$-Euler polynomials of order $k$ by using the $p$-adic $q$-integral and some transformation techniques. In particular, he constructed a new complex $q$-analogue of twisted Lerch type Euler zeta function at negative integers which interpolate the above twisted $q$-Euler polynomials.

The aim of the present note is to perform a further investigation for the $q$-extension of the twisted Lerch Euler zeta functions considered by Jang [5]. By using some elementary methods and techniques, we derive the generalized multiplication theorem for the $q$-extension of the twisted Lerch Euler zeta functions. It turns out that some well-known results, for example, Jang [5], Kim [9], etc., are reobtained.

\section{The restatement of results}

We firstly recall the $q$-extension of the twisted Lerch Euler zeta functions which is given by (see [5])

$$
\zeta_{q, E, \xi}(s, x)=[2]_{q} \sum_{n=0}^{\infty} \frac{(-1)^{n} \xi^{n} q^{n s}}{[x+n]_{q}^{s}},
$$

where $q, s \in \mathbb{C}$ with $|q|<1$ and $\operatorname{Re}(s)>1, \xi \in \mathbb{T}_{p}$ and $x$ is a positive real number. Obviously, the case $\xi=1$ in (2.1) leads to the $q$-extension of Hurwitz's type Euler zeta function due to Kim [11]. Now, let $a, b$ be positive integers and $j$ be a non-negative integer. If substituting $b x+b j / a$ for $x$ in $(2.1)$, we have

$$
\zeta_{q, E, \xi}\left(s, b x+\frac{b j}{a}\right)=[2]_{q} \sum_{n=0}^{\infty} \frac{(-1)^{n} \xi^{n} q^{n s}}{[b x+b j / a+n]_{q}^{s}}
$$

It is easy to see that for any complex numbers $x$ and $y,[x y]_{q}=[x]_{q}[y]_{q^{x}}$. Hence, in view of replacing $q$ by $q^{a}$ and $\xi$ by $\xi^{a}$ in (2.2), we derive

$$
\begin{aligned}
\zeta_{q^{a}, E, \xi^{a}}\left(s, b x+\frac{b j}{a}\right) & =[2]_{q^{a}} \sum_{n=0}^{\infty} \frac{(-1)^{n} \xi^{a n} q^{a n s}}{[b x+b j / a+n]_{q^{a}}^{s}} \\
& =[2]_{q^{a}}[a]_{q}^{s} \sum_{n=0}^{\infty} \frac{(-1)^{n} \xi^{a n} q^{a n s}}{[a b x+b j+a n]_{q}^{s}} .
\end{aligned}
$$

Since for any non-negative integer $n$ and positive integer $b$, there exist unique non-negative integers $r$ and $i$ such that $n=b r+i$ with $0 \leq i \leq b-1$. So the 
above identity (2.3) can be rewritten as follows

$$
\zeta_{q^{a}, E, \xi^{a}}\left(s, b x+\frac{b j}{a}\right)=[2]_{q^{a}}[a]_{q}^{s} \sum_{i=0}^{b-1} \sum_{n=0}^{\infty} \frac{(-1)^{b n+i} \xi^{a(b n+i)} q^{a s(b n+i)}}{[a b x+b j+a(b n+i)]_{q}^{s}} .
$$

It follows from (2.4) that

$$
\begin{aligned}
& \frac{[b]_{q}^{s}}{[2]_{q^{a}}} \sum_{j=0}^{a-1}(-1)^{j} \xi^{b j} q^{b s j} \zeta_{q^{a}, E, \xi^{a}}\left(s, b x+\frac{b j}{a}\right) \\
= & \left([a]_{q}[b]_{q}\right)^{s} \sum_{j=0}^{a-1}(-1)^{j} \xi^{b j} q^{b j s} \sum_{i=0}^{b-1}(-1)^{i} \xi^{a i} q^{a i s} \sum_{n=0}^{\infty} \frac{(-1)^{b n} \xi^{a b n} q^{a b n s}}{[a b(x+n)+a i+b j]_{q}^{s}}
\end{aligned}
$$

In the same way,

$$
\begin{aligned}
& \frac{[a]_{q}^{s}}{[2]_{q^{b}}} \sum_{j=0}^{b-1}(-1)^{j} \xi^{a j} q^{a s j} \zeta_{q^{b}, E, \xi^{b}}\left(s, a x+\frac{a j}{b}\right) \\
= & \left([a]_{q}[b]_{q}\right)^{s} \sum_{j=0}^{b-1}(-1)^{j} \xi^{a j} q^{a j s} \sum_{i=0}^{a-1}(-1)^{i} \xi^{b i} q^{b i s} \sum_{n=0}^{\infty} \frac{(-1)^{a n} \xi^{a b n} q^{a b n s}}{[a b(x+n)+b i+a j]_{q}^{s}}
\end{aligned}
$$

Thus, if $a$ and $b$ in $(2.5)$ and (2.6) satisfy $a \equiv b(\bmod 2)$, then we immediately obtain:

Theorem 2.1. Let $s, q \in \mathbb{C}$ with $|q|<1$. Then for positive integers $a$ and $b$ with the same parity,

$$
\begin{aligned}
& \frac{[b]_{q}^{s}}{[2]_{q^{a}}} \sum_{j=0}^{a-1}(-1)^{j} \xi^{b j} q^{b s j} \zeta_{q^{a}, E, \xi^{a}}\left(s, b x+\frac{b j}{a}\right) \\
= & \frac{[a]_{q}^{s}}{[2]_{q^{b}}} \sum_{j=0}^{b-1}(-1)^{j} \xi^{a j} q^{a s j} \zeta_{q^{b}, E, \xi^{b}}\left(s, a x+\frac{a j}{b}\right) .
\end{aligned}
$$

Next, we discuss some special cases of Theorem 2.1. Setting $b=1$ in Theorem 2.1, we have the following distribution formula

$$
\zeta_{q, E, \xi}(s, a x)=\frac{[2]_{q}}{[2]_{q^{a}}[a]_{q}^{s}} \sum_{j=0}^{a-1}(-1)^{j} \xi^{j} q^{s j} \zeta_{q^{a}, E, \xi^{a}}\left(s, x+\frac{j}{a}\right) .
$$

Especially, setting $a=2$ in (2.8), we have the duplication formula

$$
\zeta_{q, E, \xi}(s, 2 x)=\frac{1}{[2]_{q^{2}}[2]_{q}^{s-1}}\left(\zeta_{q^{2}, E, \xi^{2}}(s, x)-\xi q^{s} \zeta_{q^{2}, E, \xi^{2}}\left(s, x+\frac{1}{2}\right)\right) .
$$

On the other hand, since the twisted $q$-Euler polynomials can be expressed in following way (see [5, Theorem 4])

$$
E_{m, q, \xi}^{(-m, 1)}(x)=[2]_{q} \sum_{n=0}^{\infty}(-1)^{n} q^{-m n} \xi^{n}[x+n]_{q}^{m},
$$


then by $(2.1),(2.10)$ and the analytic continuation of $\zeta_{q, E, \xi}(s, x)$, one can easily obtain

$$
E_{m, q, \xi}^{(-m, 1)}(x)=\zeta_{q, E, \xi}(-m, x)
$$

In fact, using the relation

$$
[x+n]_{q}^{m}=\frac{1}{(1-q)^{m}} \sum_{i=0}^{m}\left(\begin{array}{c}
m \\
i
\end{array}\right)(-1)^{i} q^{(x+n) i},
$$

the above identity (2.10) can be reduced in the following way

$$
E_{m, q, \xi}^{(-m, 1)}(x)=\frac{[2]_{q}}{(1-q)^{m}} \sum_{i=0}^{m}\left(\begin{array}{c}
m \\
i
\end{array}\right)(-1)^{i} \frac{q^{x i}}{1+\xi q^{i-m}},
$$

which means the symmetric distribution of the twisted $q$-Euler polynomials

$$
E_{m, q, \xi}^{(-m, 1)}(x)=(-1)^{m+1} q \xi E_{m, q^{-1}, \xi^{-1}}^{(-m, 1)}(1-x) .
$$

Thus, by applying (2.11) to Theorem 2.1, we state:

Theorem 2.2. Let $a, b, m$ be positive integers with $a \equiv b(\bmod 2)$. Then

$$
\begin{aligned}
& \frac{[a]_{q}^{m}}{[2]_{q^{a}}} \sum_{j=0}^{a-1}(-1)^{j} \xi^{b j} q^{-b m j} E_{m, q^{a}, \xi^{a}}^{(-m, 1)}\left(b x+\frac{b j}{a}\right) \\
= & \frac{[b]_{q}^{m}}{[2]_{q^{b}}} \sum_{j=0}^{b-1}(-1)^{j} \xi^{a j} q^{-a m j} E_{m, q^{b}, \xi^{b}}^{(-m, 1)}\left(a x+\frac{a j}{b}\right) .
\end{aligned}
$$

It follows that we show some special cases of Theorem 2.2. Setting $b=1$ and replacing $x$ by $x / a$ in Theorem 2.2 , we have the following multiplication formula of the twisted $q$-Euler polynomials due to Jang (see [5, Theorem 3])

$$
E_{m, q, \xi}^{(-m, 1)}(x)=\frac{[2]_{q}[a]_{q}^{m}}{[2]_{q^{a}}} \sum_{j=0}^{a-1}(-1)^{j} \xi^{j} q^{-m j} E_{m, q^{a}, \xi^{a}}^{(-m, 1)}\left(\frac{x+j}{a}\right) \quad(2 \nmid a) .
$$

If multiplying $\sum_{m=0}^{\infty} t^{m} / m$ ! in both sides of (2.10), one can easily derive

$$
\begin{aligned}
\sum_{m=0}^{\infty} E_{m, q, \xi}^{(-m, 1)}(x) \frac{t^{m}}{m !} & =[2]_{q} \sum_{n=0}^{\infty}(-1)^{n} \xi^{n} \sum_{m=0}^{\infty} q^{-m n}[x+n]_{q}^{m} \frac{t^{m}}{m !} \\
& =[2]_{q} \sum_{n=0}^{\infty}(-1)^{n} \xi^{n} e^{q^{-n}[x+n]_{q} t} .
\end{aligned}
$$

It follows from $(2.17)$ that

$$
\lim _{q \rightarrow 1} E_{m, q, 1}^{(-m, 1)}(x)=E_{m}(x),
$$

where $E_{n}(x)$ denotes the classical Euler polynomials given by (see $\left.[1,2,3]\right)$

$$
\frac{2 e^{x t}}{e^{t}+1}=\sum_{n=0}^{\infty} E_{n}(x) \frac{t^{n}}{n !} \quad(|t|<\pi) \text {. }
$$


Hence, by setting $\xi=1$ and letting $q \rightarrow 1$ in Theorem 2.2, we obtain that for positive integers $a, b$ and non-negative integer $n$,

$$
a^{n} \sum_{j=0}^{a-1}(-1)^{j} E_{n}\left(b x+\frac{b j}{a}\right)=b^{n} \sum_{j=0}^{b-1}(-1)^{j} E_{n}\left(a x+\frac{a j}{b}\right) \quad(a \equiv b(\bmod 2)),
$$

which was rediscovered by many authors; see for example $[14,9]$. For the generalization of (2.20) in other direction, see [15] for a detail introduction. If substituting $x+y$ for $x$ in $(2.17)$, then by using the relation $[x+y]_{q}=[x]_{q}+q^{x}[y]_{q}$ for any complex numbers $x$ and $y$, we get

$$
\sum_{m=0}^{\infty} E_{m, q, \xi}^{(-m, 1)}(x+y) \frac{t^{m}}{m !}=[2]_{q} \sum_{n=0}^{\infty}(-1)^{n} \xi^{n} e^{q^{-n}[y+n]_{q} q^{x} t} e^{q^{-n}[x]_{q} t} .
$$

Putting the exponential series $e^{x t}=\sum_{n=0}^{\infty} x^{n} t^{n} / n$ ! and (2.17) to (2.21), with help of the Cauchy product, we derive

$$
\begin{aligned}
\sum_{m=0}^{\infty} E_{m, q, \xi}^{(-m, 1)}(x+y) \frac{t^{m}}{m !} & =\left(\sum_{m=0}^{\infty}[x]_{q}^{m} \frac{t^{m}}{m !}\right)\left(\sum_{m=0}^{\infty} q^{m x} E_{m, q, q^{-m} \xi}^{(-m, 1)}(y) \frac{t^{m}}{m !}\right) \\
& =\sum_{m=0}^{\infty}\left(\sum_{i=0}^{m}\left(\begin{array}{c}
m \\
i
\end{array}\right) q^{i x} E_{i, q, q^{-i} \xi}^{(-i, 1)}(y)[x]_{q}^{m-i}\right) \frac{t^{m}}{m !}
\end{aligned}
$$

Hence, by comparing the coefficients of $t^{m} / m$ ! in $(2.22)$, we obtain the addition theorem of the twisted $q$-Euler polynomials as follows

$$
E_{m, q, \xi}^{(-m, 1)}(x+y)=\sum_{i=0}^{m}\left(\begin{array}{c}
m \\
i
\end{array}\right) q^{i x} E_{i, q, q^{-i} \xi}^{(-i, 1)}(y)[x]_{q}^{m-i} .
$$

In light of applying (2.23) to Theorem 2.2, we immediately derive after some calculation.

Theorem 2.3. Let $a, b, m$ be positive integers with $a \equiv b(\bmod 2)$. Then

$$
\begin{aligned}
& {[2]_{q^{b}} \sum_{i=0}^{m}\left(\begin{array}{c}
m \\
i
\end{array}\right)[a]_{q}^{i}[b]_{q}^{m-i} E_{i, q^{a}, q^{-i a} \xi^{a}}^{(-i, 1)}(b x) S_{m-i, \xi^{b} ; q^{b}}(a) } \\
= & {[2]_{q^{a}} \sum_{i=0}^{m}\left(\begin{array}{c}
m \\
i
\end{array}\right)[b]_{q}^{i}[a]_{q}^{m-i} E_{i, q^{b}, q^{-i b} \xi^{b}}^{(-i, 1)}(a x) S_{m-i, \xi^{a} ; q^{a}}(b), }
\end{aligned}
$$

where $S_{m, \xi ; q}(a)=\sum_{j=0}^{a-1}(-\xi)^{j} q^{-m j}[j]_{q}^{m}$.

If taking $\xi=1$ and letting $q \rightarrow 1$ in Theorem 2.3, then we have the following identity between the classical Euler polynomials and alternating sum (see [14, 9])

$$
\sum_{i=0}^{n}\left(\begin{array}{c}
n \\
i
\end{array}\right) a^{n-i} b^{i} E_{n-i}(b x) S_{i}(a)=\sum_{i=0}^{m}\left(\begin{array}{c}
n \\
i
\end{array}\right) b^{n-i} a^{i} E_{n-i}(a x) S_{i}(b),
$$


where $n$ is a non-negative integer, $a, b$ are positive integers with $a \equiv b(\bmod 2)$ and $S_{n}(a)=\sum_{j=0}^{a-1}(-1)^{j} j^{n}$. For the generalization of the above identity (2.25) in the Apostol-type direction, the interested readers may consult to [15].

Acknowledgement. The authors express their gratitude to the referee for his or her helpful comments and suggestions in improving this paper. This work is supported by the National Natural Science Foundation of China (Grant No. 11071194).

\section{References}

[1] Y. He and Q. Y. Liao, Some congruences invloving Euler numbers, Fibonacci Quart. 46/47 (2008/2009), 225-234.

[2] Y. He and W. P. Zhang, Some symmetric identities involving a sequence of polynomials, Electron. J. Combin. 17 (2010), no. 1, Note 7, 7 pp.

[3] forms Spec. Funct. 22 (2011), no. 3, 207-215.

[4] L. Jang, On a q-analogue of the p-adic generalized twisted L-functions and p-adic $q$ integrals, J. Korean Math. Soc. 44 (2007), no. 1, 1-10.

[5] Soc. 47 (2010), no. 6, 1181-1188.

[6] T. Kim, q-Volkenborn integration, Russ. J. Math. Phys. 9 (2002), no. 3, 288-299.

[7] _ , q-generalized Euler numbers and polynomials, Russ. J. Math. Phys. 13 (2006), no. 3, 293-298.

[8] — On the analogs of Euler numbers and polynomials associated with p-adic qintegral on $\mathbb{Z}_{p}$ at $q=-1$, J. Math. Anal. Appl. 331 (2007), no. 2, 779-792.

[9] - Symmetry p-adic invariant integral on $\mathbb{Z}_{p}$ for Bernoulli and Euler polynomials, J. Difference Equ. Appl. 14 (2008), no. 12, 1267-1277.

[10] $\ldots$-adic interpolating function for $q$-Euler numbers and its derivatives, J. Math. Anal. Appl. 339 (2008), no. 1, 598-608.

[11] _ Note on the Euler q-zeta functions, J. Number Theory 129 (2009), no. 7, 17981804.

[12] _ Some identities on the q-Euler polynomials of higher order and q-Stirling umbers by the fermionic p-adic integral on $\mathbb{Z}_{p}$, Russ. J. Math. Phys. 16 (2009), no. 4, $484-491$.

[13] _ On a q-analogue of the p-adic log gamma functions and related integrals, J. Number Theory 76 (1999), no. 2, 320-329.

[14] H. M. Liu and W. P. Wang, Some identities on the Bernoulli, Euler and Genocchi polynomials via power sums and alternate power sums, Discrete Math. 309 (2009), no. 10, 3346-3363.

[15] D. Q. Lu and H. M. Srivastava, Some series identities involving the generalized Apostol type and related polynomials, Comput. Math. Appl. 62 (2011), no. 9, 3591-3602.

[16] H. Ozden and Y. Simsek, A new extension of q-Euler numbers and polynomials related to their interpolation functions, Appl. Math. Lett. 21 (2008), no. 9, 934-939.

[17] S. H. Rim and T. Kim, A note on p-adic Euler measure on $\mathbb{Z}_{p}$, Russ. J. Math. Phys. 13 (2006), no. 3, 358-361.

[18] Y. Simsek, On twisted q-Hurwitz zeta function and q-two-variable L-function, Appl. Math. Comput. 187 (2007), no. 1, 466-473.

[19] Y. Simsek, V. Kurt, and D. Kim, New approach to the complete sum of products of the twisted $(h ; q)$-Bernoulli numbers and polynomials, J. Nonlinear Math. Phys. 14 (2007), no. $1,44-56$. 


\section{YuAN He}

Department of Mathematics

Kunming University of Science and Technology

Kunming, Yunnan 650500, P. R. China

E-mail address: hyyhe@yahoo.com.cn

WenPeng Zhang

Department of Mathematics

NORTHWEST UNIVERSITY

XI'An, ShaAnXi 710127, P. R. China

E-mail address: wpzhang@nwu.edu.cn 